\title{
[gw22-e0147] CLINICAL OUTCOMES OF PATIENTS WITH HEART FAILURE AND PRESERVED EJECTION FRACTION IN CHINESE
}

Li Longgui,' Geng Zhaohua,, Zhao Xiaohui, ' Li Hongmei, ${ }^{2}$ Dang Tao' ${ }^{1}$ Xinqiao Hospital; 2323 Hospital

\subsection{6/heartjnl-2011-300867.633}

Background Although heart failure is recognised as a major health problem, little is known about heart failure with preserved ejection fraction (HFPEF) in the Chinese population. Therefore, we observed the clinical characteristics and outcomes of patients with HFPEF in China.

Methods All patients admitted with heart failure were divided into the HFPEF ( $\mathrm{EF}^{3} 50 \%$ ) and reduced ejection fraction (HFREF, EF $<50 \%$ ) groups. EF was determined by echocardiography, and clinical data were collected from a secure webbased system.

Results Out of the 534 patients with heart failure, 269 (50.4\%) had HFPEF. Compared to patients with HFREF, patients with HFPEF were older, were more likely to be female and had a higher prevalence of hypertension. The in-hospital mortality of patients with HFPEF (3.7\%) was comparable to that of patients with HFREF (4.5\%). After a mean follow-up period of 321 days, the survival of patients with HFPEF (12.7\%) was significantly better than that of patients with HFREF $(23.3 \%$, $\mathrm{p}=0.02)$. Cox proportional hazard regression identified serum creatinine levels (HR 1.006, 95\% CI 1.001 to $1.009, p=0.009$ ) and hypertension (HR 2.104, 95\% CI 1.016 to 4.359, $\mathrm{p}=0.045$ ) as predictors of survival for patients with HFPEF.

Conclusions HFPEF is common in China. Although the inhospital mortality of patients with HFPEF was comparable to that of patients with HFREF, the survival of patients with HFPEF was significantly better than that of patients with HFREF during a follow-up period lasting more than half a year after discharge. 